\title{
Influence of the static magnetic field on cell response in a miniaturized optically accessible bioreactor for 3D cell culture
}

\author{
Luca Izzo ${ }^{1}$ (1) Marta Tunesi $^{1} \cdot$ Lucia Boeri $^{1} \cdot$ Matteo Laganà $^{2} \cdot$ Carmen Giordano $^{1} \cdot$ Manuela Teresa Raimondi $^{1}$
}

Published online: 13 March 2019

(C) The Author(s) 2019

\begin{abstract}
Hydraulic sealing is a crucial condition for the maintenance of sterility during long term operation of microfluidic bioreactors. We developed a miniaturized optically accessible bioreactor (MOAB) allowing perfused culture of 3D cellularised constructs. In the $\mathrm{MOAB}$, the culture chambers are sealed by magnets that generate a weak static magnetic field (SMF). Here, we predicted computationally the exact level of SMF to which cells are subjected during culture in the MOAB and we assessed its influence on the viability, metabolic activity and gene expression of neuroblastoma-derived cells cultured up to seven days. The predicted SMF ranged from 0.32 to $0.57 \mathrm{~T}$ using an axial-symmetric model of a single chamber, whereas it ranged from 0.35 to $0.62 \mathrm{~T}$ using a $3 \mathrm{D}$ model of the complete device. Cell function was evaluated in SH-SY5Y neuroblastoma cells at 2 and 7 days of culture in the MOAB, compared to 2D monolayer, 3D non-perfused constructs, and 3D perfused constructs cultured in a modified MOAB with magnet-free sealing. We measured the cell metabolic activity normalized by the DNA content and the expression levels of heat-shock protein 70 (Hsp-70), Bcl-2 and Bax. We found that the level of SMF applied to cells in the MOAB did not influence their metabolic activity and exerted a stressful effect in 2D monolayer, not confirmed in 3D conditions, neither static not perfused. Instead, the magnets provided a significantly greater hydraulic sealing in long-term culture, thus the MOAB might be potentially exploitable for the development of reliable in vitro models of neurodegeneration.
\end{abstract}

Keywords MOAB $\cdot$ Static magnetic field $\cdot$ Perfusion bioreactors $\cdot$ Numerical characterization $\cdot$ 3D cell culture $\cdot$ Cell metabolic activity $\cdot$ Gene expression

\section{Introduction}

In vitro cell culture on two-dimensional (2D) glass or plastic substrates fails to model the complex physiological three-dimensional (3D) environment, enable cell differentiation and reproduce in vivo cell behavior; furthermore, the

Luca Izzo and Marta Tunesi contributed equally to this work

Electronic supplementary material The online version of this article (https://doi.org/10.1007/s10544-019-0387-8) contains supplementary material, which is available to authorized users.

Luca Izzo

luca.izzo@polimi.it

1 Department of Chemistry, Materials and Chemical Engineering " $\mathrm{G}$. Natta", Politecnico di Milano, Piazza Leonardo da Vinci 32, 20133 Milan, Italy

2 Gemma Prototipi Studio, Erba, Italy absence of a continuous flow of medium does not provide a continuous nutrient supply and waste removal ( $\mathrm{Lv}$ et al. 2017). Oppositely, in vitro cell modelling with miniaturized bioreactors shows great advantages. It requires small volumes of reagents and cells, both critical elements for valuable samples and high-throughput screening. Portability, design versatility, potential for parallel operations and integration with existing devices or platforms are further advantages (Lübberstedt et al. 2015). For instance, Lei and colleagues (Lei et al. 2014) provided an interesting example of a microfluidic chip coupling both 3D microenvironment and perfusion by perfusing human oral cancer cells embedded in an agarose gel at a flow rate of $10 \mu \mathrm{L} / \mathrm{h}$ in a culture chamber of $4 \times 2 \times 1 \mathrm{~mm}$.

Optical accessibility represents a considerable improvement, since it allows for the reduction of samples and the analysis of the same constructs with time by non-destructive techniques like viable staining and standard fluorescence microscopy. As an example, Kim and co-workers (Kim et al. 
2012) applied a miniaturized optically accessible perfusion bioreactor with the possibility to host $3 \mathrm{D}$ tissues for in vitro modelling of the human gut.

In this context, Laganà and Raimondi (2012) developed a miniaturized optically accessible bioreactor (MOAB) for the interstitial perfusion of $3 \mathrm{D}$ cell constructs. To simplify the assembly procedure while reducing the time required, we have optimized their initial prototype. The current device (Fig. 1, top) is composed of three independent and magnetically lockable chambers $\left(9 \mathrm{~mm}^{3}\right)$ assembled on the top surface of a common main body $(68 \times 25 \mathrm{~mm})$ made up of medical grade polystyrene. The main body has rigid edges to reduce the optical path and enhance sample illumination during optical transmission microscopy. The first magnet ( $\mathrm{NdFeB}$ ring magnet, $12 \mathrm{~mm}$ outer diameter, $9 \mathrm{~mm}$ internal diameter, $1.5 \mathrm{~mm}$ thick) is located in the chamber, while the second one is in the bioreactor body. Their magnetic coupling (closure force of $14.7 \mathrm{~N}$ ) ensures the hydraulic sealing during the perfusion of $3 \mathrm{D}$ constructs $(6 \times 3 \times 0.4 \mathrm{~mm})$ and simplifies the assembly procedure, allowing for the self-centering and self-aligning of the locking system with respect to the feed channels. Each chamber has a $9 \mathrm{~mm}$ glass coverslip equipped with a medical grade silicone gasket. It prevents the movements of the constructs by mechanical interference. By changing the shape of the gasket, it is possible to host differently shaped constructs (e.g. scaffolds, hydrogels) in the culture chambers. The whole device may be sterilized with hydrogen peroxide gas plasma systems (e.g. STERRAD® 100S, ASP, Jonhson \& Jonhson, Irvine, CA, USA). The MOAB is a versatile and tunable platform, already validated for advanced in vitro cell modelling in several research fields, including neuroscience (Tunesi et al. 2016) and cancer (Marturano-Kruik et al. 2018). Thanks to its optical accessibility, it was also applied for in vitro tracking of exosomes in a model of gene therapy for muscular dystrophy (Frattini et al. 2017).

The magnets sealing the culture chambers of the MOAB generate a static magnetic field (SMF) that might influence cell functions. SMFs interact with biological systems by both electrodynamic and magneto-mechanical effects, meaning that moving ionic charges induce an electric potential because of the Lorentz force and both diamagnetic and magnetic substances are exposed to a torque and orient, respectively (Roth 2011). SMFs are naturally present: the Earth itself generates a magnetic field ranging between 25 and $65 \mu \mathrm{T}$ and involved in a.

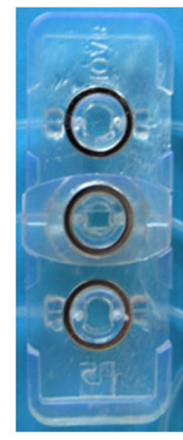

e.

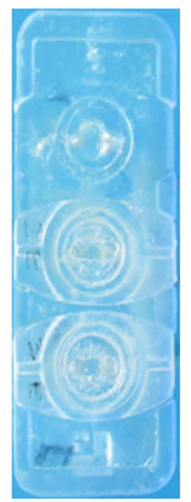

b.

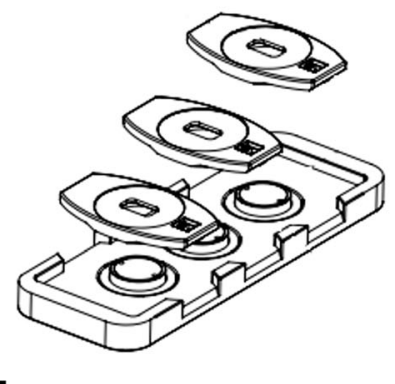

f.

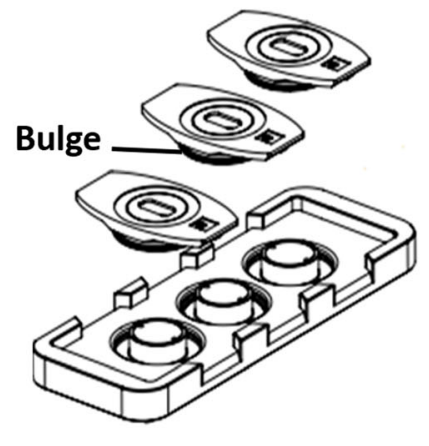

Fig. 1 Views and technical sketches (AutoCAD ${ }^{\circledR}$ Software, Autodesk, San Rafael, California, USA) of the miniaturized optically accessible bioreactor (MOAB) with magnetic (upper panel) or snap-fit closure (lower panel). a Top view of the MOAB with magnetic closure. b Technical sketch showing the MOAB with magnetic closure. c Top: top view of one culture chamber with magnetic closure; Bottom: side sketch of one culture chamber with magnetic closure. $\mathbf{d}$ Technical sketch showing the lid (green), the gasket

c.
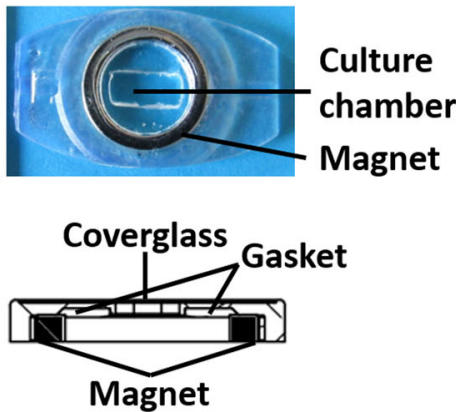

g.

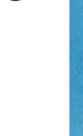
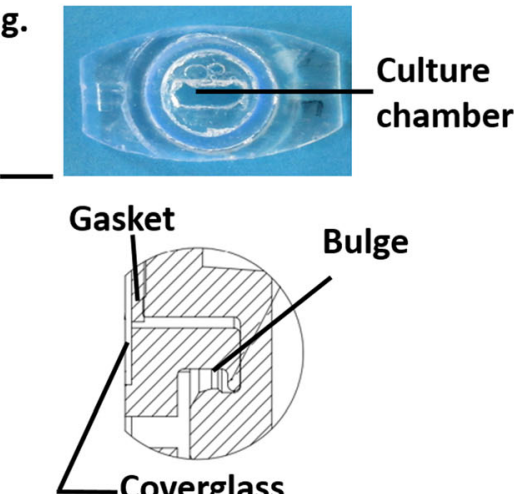

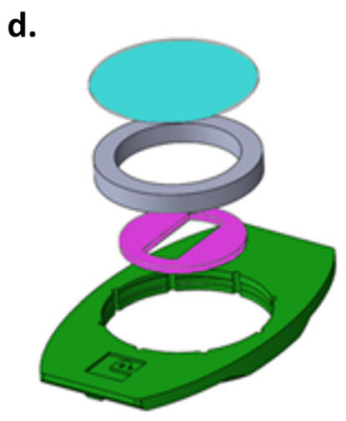

h.
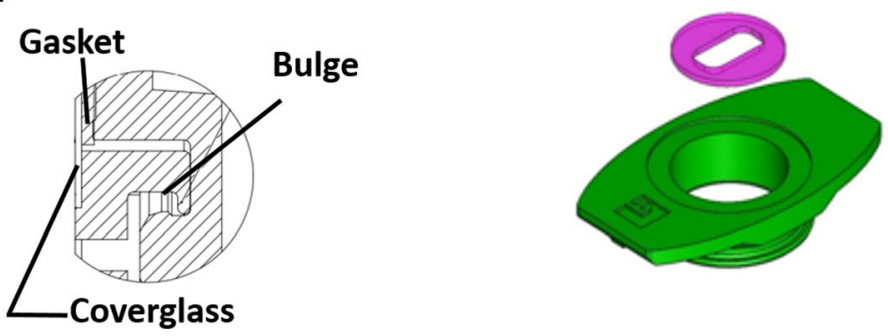

(purple), the magnet (grey) and the glass coverslip (light blue). e Top view of the MOAB without magnetic closure. $f$ Technical sketch showing the MOAB without magnetic closure. $\mathbf{g}$ Top: top view of one culture chamber without magnetic closure; Bottom: side sketch of one culture chamber without magnetic closure. $\mathbf{h}$ Technical sketch showing the lid (green), the gasket (purple) and the glass coverslip (light blue) in the MOAB without magnetic closure. Scale bar $5 \mathrm{~mm}$ 
the orientation and migration of some animal species (Feychting 2005). Industrial activities exploiting direct currents also generate SMFs (e.g. in aluminum production or chloralkali plants, workers are exposed to SMFs varying from 4 to $50 \mathrm{mT}$ (European Commission 1996)). Therapeutic devices (e.g. prosthetic cardiac pacemaker, defibrillator, standard orthodontic devices, Holter) also produce SMFs up to $10 \mathrm{mT}$. In particular, defibrillators and standard orthodontic devices expose the patients to a SMF for very short periods, but the operators for frequent times. For medical and research purposes, stronger SMFs may be applied. For instance, for magnetic resonance imaging (MRI) the magnetic field can reach intensities up to $3 \mathrm{~T}$ (Hartwig et al. 2018), while the operators are exposed to a magnetic field varying from 0.5 to $2 \mathrm{mT}$ (Zannella 1997).

Valiron and colleagues (Valiron et al. 2005) observed that exposure to high magnetic fields (over $10 \mathrm{~T}$ and $15 \mathrm{~T}$ for cycling cells and neurons, respectively) for 30-60 min affects cell cytoskeleton, with deleterious effects on cell viability (e.g. detachment from culture dishes), organization and differentiation. A reduction in cell viability was also reported when decreasing the intensity of the SMF and increasing the exposure time. For example, Raylman and co-workers (Raylman et al. 1996) obtained a reduction in the number of viable HTB 63, HTB 77 IP3 and CCL 86 malignant human cell lines placed for $64 \mathrm{~h}$ in the isocenter ( $\sim 5 \mathrm{~cm}$ diameter) of a superconducting solenoid magnet generating a $7 \mathrm{~T}$ uniform SMF. Similarly, Ji and colleagues (Ji et al. 2009) observed a decrease in the number of $E$. coli colony-forming units after exposures up to $60 \mathrm{~min}$ to two different permanent magnets (magnetic field induction between 45 and $450 \mathrm{mT}$ and $0.45-3.5 \mathrm{~T}$. In the latter configuration, the maximum space for exposure was $17 \times 17 \times 10 \mathrm{~cm}$ ). To study the effects of the intensity of the SMF that patients are exposed to during MRI, Zhang and co-workers (Zhang et al. 2017) plated 15 different cell lines on the top surface of a magnet $(1 \mathrm{~T}, 5 \times 5 \times 5$ $\mathrm{cm})$, reporting that the SMF affects cell proliferation in a cell type- and density-dependent manner. The dependence on cell type was also suggested by Aldinucci and colleagues (Aldinucci et al. 2003), showing that the combination of a static electromagnetic field $(4.75 \mathrm{~T})$ with a pulsed electromagnetic field $(0.7 \mathrm{mT})$ generated by an NMR apparatus had neither proliferative, nor activating or proinflammatory effect on lymphocytes after $1 \mathrm{~h}$-exposure, while it affected proliferation in Jurkat cells.

This work takes place inside two novel technological projects, named MOAB (ID number 825159) and MINERVA (ID number 724734) supported by the European Research Council (ERC) Programme. In particular, MINERVA project aims, by using an innovative bioengineering approach, at evaluating the potential impact of human gut microbial community on central nervous system functionality also in neurodegenerative disorders. MINERVA goal is to develop a cutting-edge technological platform, based on organ on chip microfluidic device, to model the main players of the microbiota-brain axis connection. In this context, with a view to exploit the improved version of the MOAB also as the basic functional unit for the development of reliable in vitro models of neurodegeneration in MINERVA project, in this work we focused on predicting the SMF generated by its magnets and measuring its effects on viability, metabolic activity and gene expression in SH-SY5Y neuroblastoma cells, a commonly used cell model in the research against neurodegeneration. As a control, we used MOABs with a non-magnetic lock (e.g. snap-fit closure, Fig. 1, bottom), a custom-made version of the device where the magnets are not present and the hydraulic sealing is assured by the mechanical interference between the lids (showing a bulge) and the main body of the bioreactor.

\section{Materials and methods}

\subsection{Numerical prediction of the level of magnetic field}

To study the distribution of the intensity of the SMF generated by the magnetic closures, we performed a numerical analysis with the software COMSOL Multiphysics ${ }^{\circledR}$ (Burlington, MA, USA), version 5.2. We exploited both an axial-symmetric and a $3 \mathrm{D}$ complete model and compared their results. In the first model, we considered only one chamber (thus hypothesizing that they are independent), while in the second one we included all the three chambers (thus taking into account their possible reciprocal influences).

The geometry of the 3D complete model (Fig. 2) was represented by three couples of permanent ring magnets (Supermagnete, Gottmadingen, Germany) identical to those in the MOAB. We reported their properties in Table 1. We chose neodymium as the material for the domains of magnetic coupling. To simulate the external environment and visualize the distribution of the magnetic field intensity around the chambers, we assumed an air-made cylinder of $50 \mathrm{~mm}$ in diameter and $50 \mathrm{~mm}$ in height, with a magnetic insulation for the external surface. Its isocenter corresponded to the center of the central magnetic chamber; while the centers of the two lateral chambers were $16 \mathrm{~mm}$ far. This distance was in accordance with the geometry of the MOAB. We chose the dimensions of the cylinder to observe a null value of magnetic field intensity on its boundaries.

The properties of neodymium and air were already present in COMSOL Multiphysics ${ }^{\circledR}$ database, except for the relative permeability.

For both models, we inserted a value of 1.04 and 1 for neodymium and air, respectively. In addition, the Maxwell 


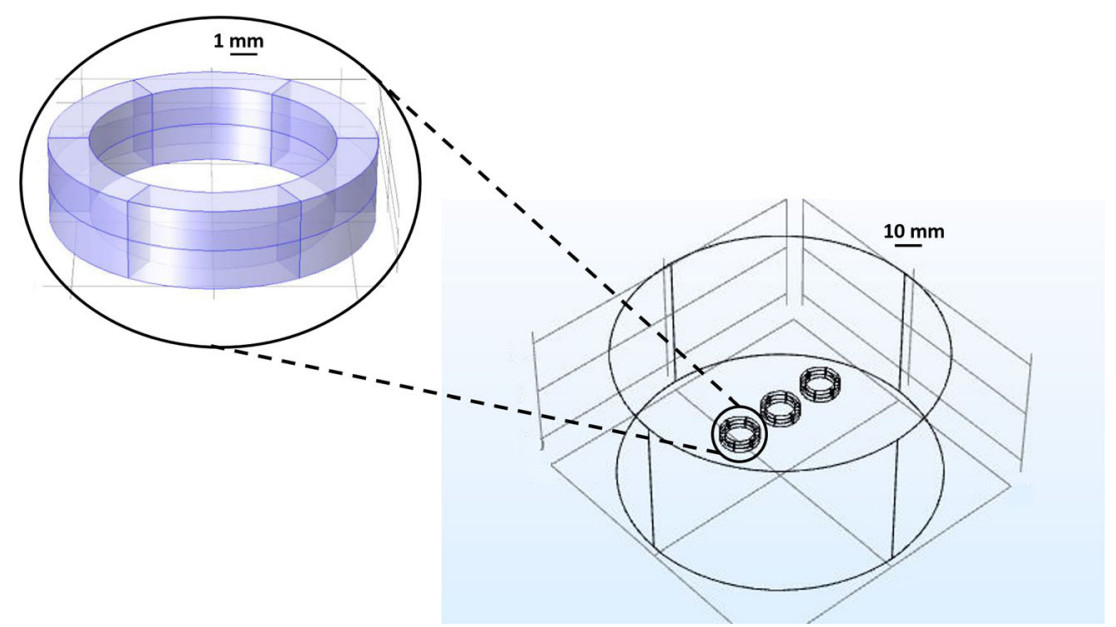

Fig. 2 COMSOL Multiphysics ${ }^{\circledR}$ modeling of the magnetic coupling of the MOAB. Geometry of the COMSOL Multiphysics ${ }^{\circledR}$ models for the three chambers of the MOAB. Left: Enlargement of a single couple of magnets and geometry of the exposure zone. Right: To visualize the distribution of the intensity of the static magnetic field, we assumed an

equations modelling the physical phenomenon were already present in the tool Magnetic Fields No Currents:

$\mathrm{H}=-\nabla \mathrm{V}_{\mathrm{m}}$

where $\mathrm{H}$ is the magnetic field intensity and $\mathrm{V}_{\mathrm{m}}$ is the magnetic scalar potential;

$\nabla \cdot\left(\mu_{0} \mu_{\mathrm{r}} \mathrm{H}+\mathrm{B}_{\mathrm{r}}\right)=0$

where $\mu_{0}$ is the vacuum permeability, $\mu_{\mathrm{r}}$ is the relative permeability of the material and $\mathrm{B}_{\mathrm{r}}$ is the residual magnetism (assumed $2.74 \mathrm{~T}$, that is $1.37 \mathrm{~T}$ multiplied by two, since we considered two coupled magnets). Other input parameters

Table 1 Properties of NdFeB magnets

\begin{tabular}{ll}
\hline Property & Value \\
\hline Material & NdFeB \\
Cross section shape & Rectangular \\
Outer diameter & $12 \mathrm{~mm}$ \\
Internal diameter & $9 \mathrm{~mm}$ \\
Height & $1.5 \mathrm{~mm}$ \\
Tolerance & $\pm 0.1 \mathrm{~mm}$ \\
Closure force & $14.7 \mathrm{~N}$ \\
Direction of magnetization & $\mathrm{AXIAL}$ (that is parallel to the height) \\
Coating & $\mathrm{Ni}-\mathrm{Cu}-\mathrm{Ni}$ \\
Magnetization & $\mathrm{N} 45$ \\
Weight & $0.56 \mathrm{~g}$ \\
Residual magnetism & $1.37 \mathrm{~T}$ \\
Max temperature & $80{ }^{\circ} \mathrm{C}$ \\
\hline
\end{tabular}

List of the features of each ring magnet (Supermagnete, Gottmadingen, Germany) integrated in the microfluidic optically accessible bioreactor (MOAB) with magnetic closure air-made cylinder with two coupled neodymium-based ring magnets in the isocenter (axial-symmetric model) or six coupled neodymium-based ring magnets (two in the isocenter and four $16 \mathrm{~mm}$ far, 3D complete model)

assumed were the temperature $(293.15 \mathrm{~K})$ and the absolute pressure (1 atm). An axially magnetized magnetic couple was present in the problem; therefore, we selected the correct domains to align the magnetized faces perpendicularly to the axial direction. We also assumed absence of radial magnetization. To estimate the intensity of magnetic induction field as a function of the distance from the symmetry axis of the magnet(s), for both models we chose the option very dense mesh for both the magnet(s) and the air-made cylinder. We extracted the results of both models in a colour map showing the distribution of the magnetic induction field around the magnet(s).

\subsection{Hydraulic characterization of magnetically and non-magnetically lockable MOABs}

\subsubsection{Static leakage tests}

To assess the capability of both magnetically and snapfit-lockable MOABs to face a pressure increase, we performed static leakage tests. We used a pressure regulator to provide pressure steps of $0.02 \mathrm{MPa}$ to a distilled water reservoir connected to the inlet channel of each culture chamber by gas permeable tubes (internal diameter: 0.03", outer diameter: 0.065"; Silastic ${ }^{\circledR}$ Tubing, Cole-Parmer, Vernon Hills, IL, USA). We tested the three chambers one by one, while clamping the outputs to allow for the pressure increase to the critical value. We maintained each pressure step for $30 \mathrm{~s}$. We measured the maximum pressure value when the first water drop started coming out from the examined chamber. We tested two magnetically lockable and two snap-fit lockable MOABs and repeated the test three times for each chamber. 


\subsubsection{Hydraulic resistance}

To characterize the hydraulic resistance of the fluid pathway, we used a precision pressure regulator to provide pressures from $0.02 \mathrm{MPa}$ to $0.1 \mathrm{MPa}$ (with steps of $0.02 \mathrm{MPa}$ ) to a distilled water reservoir connected to the inlet channels of the culture chambers. We connected the output channels to a reservoir placed on a precision electronic balance (AP250D, Ohaus, Greifensee, Switzerland) and tested the three chambers one by one. We maintained each pressure step for $30 \mathrm{~s}$, then we clamped the inlet channel and calculated the flow rate from the measured weight and the perfusion time. We tested two magnetically lockable and two snap-fit lockable MOABs and repeated the test three times for each input pressure value. To account for their hydraulic resistance, each chamber hosted a polystyrene scaffold (6x3x0.4 mm, 3D Biotek, Hillsborough, NJ, USA; Fig. 3, top) identical to those for cell experiments. It showed four layers of fibers (diameter: $100 \mu \mathrm{m}$, pore size: $300 \mu \mathrm{m}$ ) shifted of $150 \mu \mathrm{m}$ with respect to the adjacent one.

\subsubsection{Cell culture}

We cultured SH-SY5Y human neuroblastoma cells (Izsler, code BS TLC 232) at $37^{\circ} \mathrm{C}$ and $5 \% \mathrm{CO}_{2}$ in high-glucose Dulbecco's modified Eagle's medium supplemented with $10 \%(v / v)$ fetal bovine serum, $2 \mathrm{mM}$ L-glutamine, $100 \mathrm{U} / \mathrm{mL}$ penicillin, $0.1 \mathrm{mg} / \mathrm{mL}$ streptomycin sulfate (ThermoFisher Scientific,
Waltham, MA, USA) and split twice a week. We examined the effects of the magnetic field generated by the NdFeB ring magnets in both 2D and 3D (polystyrene scaffolds) conditions.

\subsubsection{Influence of the magnetic field on cells in 2D culture}

We studied the influence of the SMF on SH-SY5Y cells in 2D culture after both short (48 h) and longer exposures (7 days). For the first condition, we plated 93,750 cells $/ \mathrm{cm}^{2}$ in the center of $35 \mathrm{~mm}$ glass bottom dishes with $7 \mathrm{~mm}$ diameter center openings (MatTek Corporation, Ashland, MA, USA); while for the others, we plated 31,250 cells $/ \mathrm{cm}^{2}$. These concentrations allowed for having enough cells after $48 \mathrm{~h}$ and avoiding over confluence after 7 days.

Since our predictions suggested that the SMF generated by a single couple of $\mathrm{NdFeB}$ magnets is a reliable approximation of the SMF to which the cells are exposed in the culture chambers (regardless of their position in the MOAB), we performed experiments in $2 \mathrm{D}$ conditions by reproducing a single chamber in a custom-made setup, also suitable for confocal microscopy. To prevent the corrosion of the magnets when in contact with medium and in humidified atmosphere, we coated the magnets with High Temp Resin (Formlabs, Somerville, MA, USA). We disinfected the samples by $70 \%(v / v)$ ethanol, washed extensively with distilled water and sterilized by UV irradiation ( $254 \mathrm{~nm}$ ) for $1 \mathrm{~h}$. One day after seeding, we placed our setup in the center of the cell-seeded glass bottom dishes.
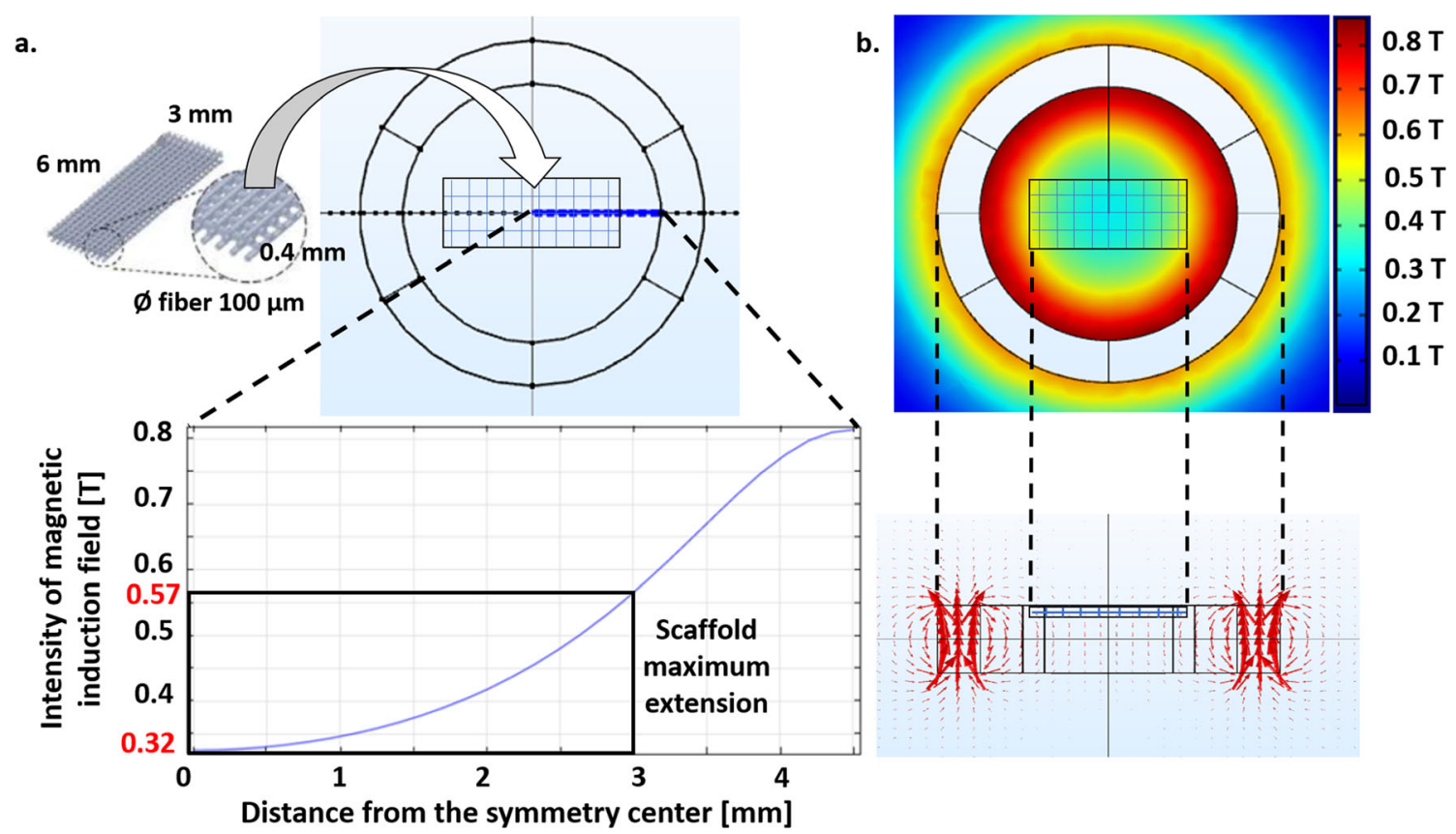

Fig. 3 Axial-symmetric model of the magnetic field generated in a single culture chamber. a Intensity of magnetic induction field with the distance from the symmetry center of the magnets (blue line). The black rectangle highlights the dimensions of polystyrene scaffolds $(6 \times 3 \times 0.4 \mathrm{~mm})$ identical to those for cell experiments. They are obtained by fused deposition modeling and composed of four layers of fibers (diameter: $100 \mu \mathrm{m}$, with a pore size: $300 \mu \mathrm{m}$ ) shifted of $150 \mu \mathrm{m}$ with respect to the adjacent. A sketch of the positioning of the scaffolds in the culture chambers is also shown. b Color map showing the distribution of the magnetic induction field around the magnets (top view) and side view of the arrow field. A sketch of the positioning of the scaffolds in the culture chambers is reported 
As a control, we cultured SH-SY5Y cells in the absence of $\mathrm{NdFeB}$ magnets in the same glass bottom dishes.

After $48 \mathrm{~h}$ and 7 days, we evaluated cell metabolic activity by resazurin (Sigma-Aldrich, St. Louis, MO, USA) assay by measuring the fluorescence at $590 \mathrm{~nm}$ (excitation wavelength 560 nm, Infinite M200PRO, Tecan, Männedorf, Switzerland). At the end of this analysis, we assessed the number of viable SH-SY5Y cells in the samples. We detached the cells from culture plates by trypsin, diluted with Trypan blue dye and counted. For each condition, we counted at least four squares. Starting from a previous study reporting that every human diploid cell contains about 6.4 pg DNA (Dolezel et al. 2003), we exploited these results to calculate the total content of DNA and the specific metabolic activity (that is cell metabolic activity normalized to the DNA content of each sample).

On day 7, we performed the live/dead assay (ThermoFisher Scientific). Medium was removed, and cells were washed with phosphate-buffered saline before incubation with $0.5 \mu \mathrm{M}$ ethidium homodimer- 1 and $0.4 \mu \mathrm{M}$ calcein AM. Finally, samples were imaged by a confocal microscope (Fluoview FV10i, Olympus, Tokyo, Japan).

On day 7, we also evaluated the effect of the SMF on these target genes: Heat Shock Protein 70 (Hsp-70), Bcl-2 and Bax. Hsp-70 is an anti-apoptotic molecule that regulates several steps of the apoptotic cascade (Kong et al. 2016), while Bcl2 has a role in inhibiting cell death. Oppositely, Bax is a proapoptotic molecule (Pawlowski and Kraft 2000). We performed the analyses on samples previously subjected to resazurin assay. We extracted the total cellular RNA with miRNeasy Mini Kit (QiazolTM, Qiagen, Hilden, Germany), according to the manufacturer instructions. We evaluated the quality of the extracted RNA via spectrophotometry (ND1000; NanoDrop ${ }^{\mathrm{TM}}$, ThermoFisher Scientific). We synthesized the cDNA starting from $500 \mathrm{ng}$ of total RNA with the High-Capacity cDNA Reverse Transcription Kit (Applied Biosystems $^{\mathrm{TM}}$, ThermoFisher Scientific). We used the cDNA to measure the relative mRNA expression levels of Hsp-70, Bcl-2 and Bax via quantitative real-time polymerase chain reaction (qRT-PCR) with TaqMan ${ }^{\mathrm{TM}}$ Reagents (Applied Biosystems $^{\mathrm{TM}}$ ). We used $18 \mathrm{~s}$ rRNA as a reference gene. For each sample, we normalized the expression of target genes to the expression level of $18 \mathrm{~s}$ rRNA. Among the groups, we normalized the expression ratio of magnetic samples to the corresponding control.

\subsubsection{Influence of the magnetic field on cells in 3D culture}

We plated SH-SY5Y cells in polystyrene scaffolds, as reported (Tunesi et al. 2016). After seeding, we kept the scaffolds in low-attachment culture plates for 2 days, and then we evaluated the effects of the SMF on SH-SY5Y cells both in dynamic and static conditions. We examined the following situations:
1. Presence of the SMF, dynamic culture: we cultured the scaffolds for $48 \mathrm{~h}$ or 7 days in dynamic conditions (MOAB with magnetic closing, flow rate: $0.5 \mu \mathrm{L} / \mathrm{min}$; PHD ULTRA programmable syringe pump, Harvard Apparatus, Holliston, MA, USA);

2. Absence of the SMF, dynamic culture: we cultured the scaffolds for $48 \mathrm{~h}$ or 7 days in dynamic conditions in the absence of the magnetic field (bioreactor without magnetic closing, flow rate: $0.5 \mu \mathrm{L} / \mathrm{min}$ );

3. Presence of the SMF, static culture: we cultured the scaffolds for $48 \mathrm{~h}$ or 7 days in the presence of the magnetic field in static conditions (inside the custom-made setup described in the previous paragraph);

4. Absence of the SMF, static culture: we cultured the scaffolds for $48 \mathrm{~h}$ or 7 days in the absence of the magnetic field in static conditions (inside a low-attachment culture plate).

After $48 \mathrm{~h}$ and 7 days, we repeated the analyses described for $2 \mathrm{D}$ cultures. We analyzed the mRNA expression levels of Hsp-70, Bcl-2 and Bax for groups 1, 3 and 4.

\subsubsection{Statistical analysis}

We reported the results as mean \pm standard deviation (SD). We performed the statistical analysis with GraphPad Prism® software (GraphPad Software, La Jolla, CA, USA). We used twoway analysis of variance (ANOVA) followed by Tukey's multiple comparisons test for comparisons among the groups and time frames, while we used one-way ANOVA followed by Dunnett's multiple comparisons test for comparisons among the groups. The significance level was set at $p<0.05$.

\section{Results}

\subsection{Numerical prediction of the level of magnetic field}

Figure 3 and Fig. 4 show the results from the analysis with COMSOL Multiphysics ${ }^{\circledR}$ for the axial-symmetric and the 3D complete model, respectively. The black rectangle highlights the dimensions of the polystyrene scaffold within the culture chambers. For both models, according to Biot-Savart law, we observed a quadratic decay for the intensity of magnetic induction field in the area occupied by the scaffold. A magnetic field is present outside the rings, but its values rapidly reach zero while increasing the distance from the symmetry center.

With the axial-symmetric model, considering only one of the three chambers, we predicted that cell constructs are exposed to a SMF ranging from $320 \mathrm{mT}$ to $570 \mathrm{mT}$. The arrow field lines showed the two magnetic poles of the coupling and confirmed the non-zero value of the magnetic field in the center of the culture chamber. 


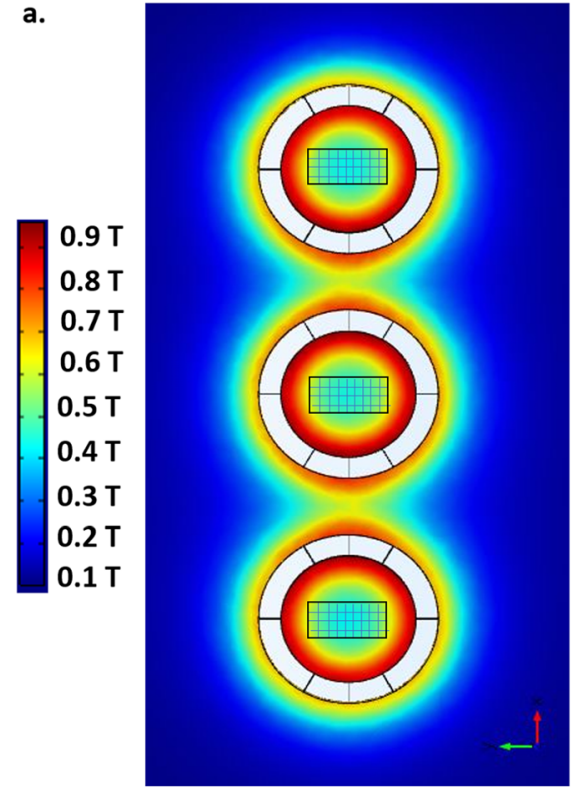

b.

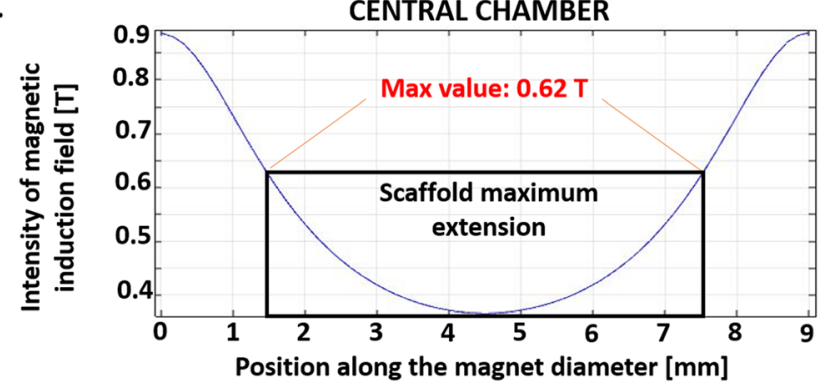

c.

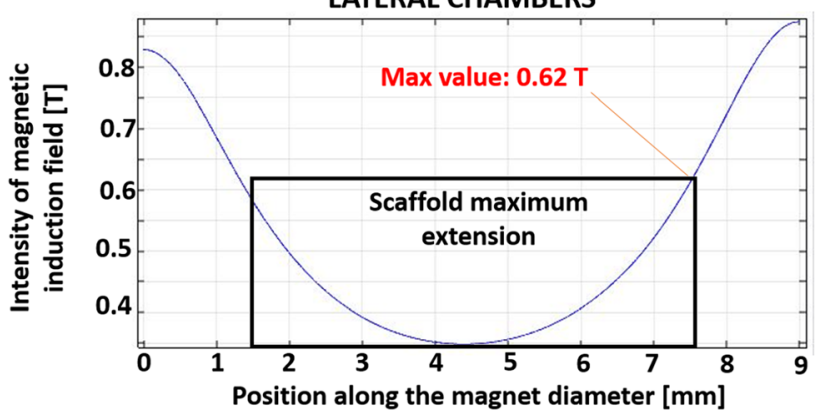

Fig. 4 Complete 3D model of the magnetic field generated by the three chambers. a Color map showing the distribution of the magnetic induction field around the magnets (top view). The black rectangles highlights the dimensions of polystyrene scaffolds. b Intensity of magnetic induction field as a function of the distance from the

With the 3D complete model, considering all the chambers and their possible reciprocal influences, we predicted that the magnetic field intensity ranges from $350 \mathrm{mT}$ to $620 \mathrm{mT}$ in the lateral chambers and from $370 \mathrm{mT}$ to $620 \mathrm{mT}$ in the central one. Therefore, when the two other chambers are included in the model, in each lateral chamber the intensity of the SMF increases by less than $10 \%$. For the central chamber, the minimum intensity of the SMF increases with respect to the lateral ones, but the maximum value is unchanged, suggesting that the influence of adjacent chambers is negligible. Therefore, a single couple of $\mathrm{NdFeB}$ magnets and an axial-symmetric model is suitable to estimate the intensity of the SMF in the scaffolds in the magnetically lockable MOABs with a reliable approximation.

Since our predictions have suggested that in the culture chambers cells are exposed to non-zero values of the SMF, effects on their viability, metabolic activity and gene expression might be observed.

\subsection{Hydraulic characterization of magnetically and non-magnetically lockable MOABs}

\subsubsection{Static leakage tests}

Figure 5a shows the results from static leakage tests. We did not find any difference (ns, $p>0.05$ ) between MOABs with magnetic and snap-fit closing, with values (mean $\pm \mathrm{SD}$ ) of $(0.110 \pm 0.017) \mathrm{MPa}$ and $(0.122 \pm 0.035) \mathrm{MPa}$, respectively. symmetry center of the magnets for the central chamber (blue line). The maximum estimated value was $620 \mathrm{mT}$. c Intensity of magnetic induction field as a function of the distance from the symmetry center of the magnets for the lateral chambers (blue line). The maximum estimated value was $620 \mathrm{mT}$

In addition, for both MOABs we did not observe breaking while increasing the pressure.

\subsubsection{Hydraulic resistance}

Figure $5 \mathrm{~b}$ shows the results related to the hydraulic resistance of the fluid pathway. For both magnetically and snap-fit lockable MOABs, the flow rate increased while raising the pressure. For a pressure of $0.02 \mathrm{MPa}$ we observed a greater flow rate (mean $\pm \mathrm{SD})$ for the snap-fit bioreactors $(1.8 \pm 0.08$ vs 3.5 $\pm 0.01 \mathrm{~mL} / \mathrm{min}, *, p<0.05)$; while for a pressure of $0.04 \mathrm{MPa}$ we did not find differences between the two configurations (ns, $p>0.05$ ). At this pressure, we recorded a flow rate of $6.0 \pm 0.01$ and $6.0 \pm 0.04 \mathrm{~mL} / \mathrm{min}$ for the magnetic and snapfit MOAB, respectively. When we further increased the pressure, the magnetically lockable bioreactor offered a greater flow rate $\left(^{* *}, p<0.01\right.$ when we set pressure at $0.06 \mathrm{MPa}$; $* * * *, p<0.0001$ when we set pressure at 0.08 and $0.1 \mathrm{MPa}$ ). At these pressures, we recorded a flow rate of $10.0 \pm 0.82$ and $8.0 \pm 0.41 \mathrm{~mL} / \mathrm{min} ; 14.0 \pm 0.82$ and $9.5 \pm$ $0.41 \mathrm{~mL} / \mathrm{min} ; 18.0 \pm 0.82$ and $14.5 \pm 0.41 \mathrm{~mL} / \mathrm{min}$ for the magnetic and snap-fit MOAB, respectively. These values are greater than those applied for cell perfusion.

\subsubsection{Influence of the magnetic field on cells in 2D culture}

Figure 6 shows the experimental set-up and the results related to the effect of the magnetic field on the viability, metabolic activity 
a.

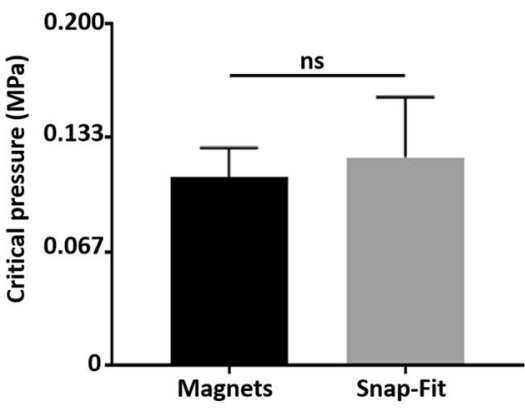

Fig. 5 Hydraulic characterization of MOABs with magnetic and snap-fit closing. a Static leakage tests. We reported the results as mean \pm SD (6 replicates/group). We performed the statistical analysis with ttest. ns, $p>0.05$. b Hydraulic resistance. We reported the results as mean

and gene expression of SH-SY5Y cells in 2D monolayer. We exposed the cells to the SMF one day after seeding because we noticed that its presence impaired cell adhesion, especially in the case of the lowest cell density $\left(31,250\right.$ cells $\left./ \mathrm{cm}^{2}\right)$.

For both metabolic activity and specific metabolic activity, we did not find differences with controls on glass substrates b.

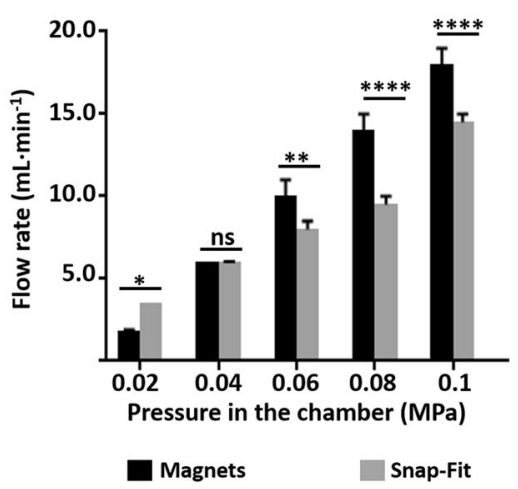

$\pm \mathrm{SD}$ (6 replicates/group). We performed the statistical analysis with oneway ANOVA followed by Tukey's multiple comparisons test. ns, $p>0.05 ; *, p<0.05 ; * *, p<0.01, * * * *, p<0.0001$

(but also on standard tissue culture-treated dishes, TCP) cultured in the absence of the magnets (ns, $p>0.05$ ), suggesting no effects of the magnetic field generated by the NdFeB magnets in the examined time window. Live/dead assay and confocal microscopy confirmed these results. Fig. 6c reports an example image: the great majority of SH-SY5Y cells cultured a.

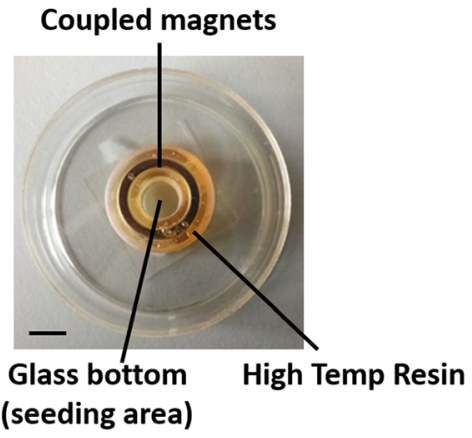

c.

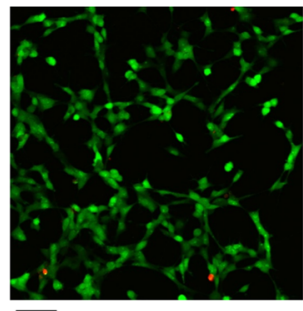

b.

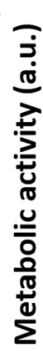

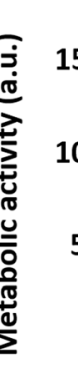

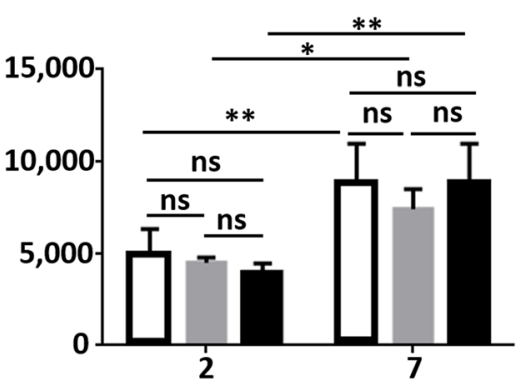

2D CONDITIONS,

d.

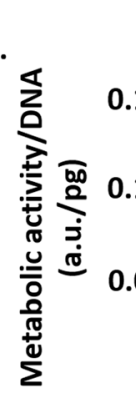

\section{$\square$ TCT Plastic \\ Glass \\ Magnets}

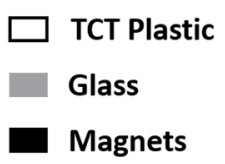

Fig. 6 Influence of the magnetic field on SH-SY5Y cells in 2D culture: specific metabolic activity. a Experimental set-up to evaluate the effect of the magnetic field generated by the ring magnets of the MOAB on SH-SY5Y cells. Scale bar: $5 \mathrm{~mm}$. b Metabolic activity of SH-SY5Y cells with time. For the short exposure time (2 days), we plated 93,750 cells $/ \mathrm{cm}^{2}$; while for the long exposure time (7 days), we plated 31,250 cells $/ \mathrm{cm}^{2}$. TCP indicates SH-SY5Y cells plated on standard tissue culture-treated dishes. $\mathbf{c}$ Example of a confocal microscopy image showing live (stained green by calcein AM) and dead (stained red by ethidium homodimer-1) SH-SY5Y cells after $48 \mathrm{~h}$ culturing in the absence of the

Exposure time (days)

Exposure time (days)

magnetic field. Scale bar: $20 \mu \mathrm{m}$. d Specific metabolic activity (that is metabolic activity normalized with respect to the DNA content) of SHSY5Y cells with time. For the shortest exposure time (2 days), we plated 93,750 cells $/ \mathrm{cm}^{2}$; while for the other exposure times, we plated 31,250 cells $/ \mathrm{cm}^{2}$. In all the graphs, we showed the results from resazurin assay (3 replicates/group). We reported the results as mean $\pm \mathrm{SD}$. We performed the statistical analysis with two-way ANOVA followed by Tukey's multiple comparisons test. ns, $p>0.05 ; *, p<0.05 ; * *, p<0.01$; ****, $p<$ 0.0001 
for 7 days in glass bottom dishes was alive (stained green by calcein AM). Only a few dead cells (stained red by ethidium homodimer-1) were visible.

Figure 7 reports the mRNA expression levels of the anti-apoptotic Hsp70 and $\mathrm{Bcl}-2$ and the pro-apoptotic Bax after 7-days exposure. It shows a lower expression of $\operatorname{Hsp} 70(*, p<0.05)$ and a higher expression of Bax $(* *, p<0.01)$ in SH-SY5Y cells exposed to the magnetic field with respect to controls on glass substrates. We did not detect significant differences in the mRNA expression levels of Bcl-2 (ns, $p>0.05$ ).

\subsubsection{Influence of the magnetic field on cells in 3D culture}

Figure 8 shows the results related to the effect of the magnetic field on the metabolic activity and specific metabolic activity of SH-SY5Y when in 3D scaffolds both in dynamic or static conditions.

After 2 days in static conditions, we did not observe effects on metabolic activity and specific metabolic activity. After 7 days, we measured a significant difference in metabolic activity $(* * *, p<0.001)$, but it was recovered when calculating the specific metabolic activity (ns, $p>0.05$ ).

In dynamic conditions, again we observed a statistical difference $(* * *, p<0.001)$ in both metabolic activity and specific metabolic activity after 7 days of exposure to the SMF. However, the situation was reversed with respect to the static condition, with the cells exposed to magnetic field exhibiting greater values than controls.

Live/dead assay and confocal microscopy supported these results. Fig. 8e shows an example image: the great majority of SH-SY5Y cells cultured for 7 days in static conditions was alive (stained green by calcein AM). Only a few dead cells (stained red by ethidium homodimer-1) may be detected. Finally, confocal microscopy suggested a good degree of scaffold colonization, reporting the presence of living cells at least on three of the four layers.

Gene expression analyses (Fig. 9) showed different Hsp$70, \mathrm{Bcl}-2$ and Bax profiles between magnetic (Mag-static conditions; MOAB-dynamic conditions) and control samples (No Mag, static conditions) with respect to SH-SY5Y cells in $2 \mathrm{D}$ conditions. The expression levels of the anti-apoptotic Hsp70 did not decrease (ns, $p>0.05$ ) and the expression levels of the pro-apoptotic Bax did not increase (ns, $p>$ 0.05 ) in magnetic samples with respect to controls. However, the expression levels of Bcl-2 were significantly lower in the presence of the SMF (**, $p<0.01$ for Mag samples; ****, $p<0.0001$ for Mag samples), suggesting a stressful effect that does not reduce cell-specific metabolic activity.

\section{Discussion}

This study aimed at assessing the effects of the SMF generated by the magnetic closure of the optimized prototype of a recently developed miniaturized bioreactor (Laganà and Raimondi 2012) on the metabolic activity, viability and gene expression of target genes (Hsp-70, Bcl-2 and Bax) in human SH-SY5Y neuroblastoma cells. Our results indicated that: 1) in the MOAB with magnetic closing, cells are exposed to non-zero values of SMF; 2) the maximum value of the SMF estimated with the $3 \mathrm{D}$ complete model $(620 \mathrm{mT})$ is independent on the chamber position (lateral or central); 3) the maximum value of the SMF estimated by the axial-symmetric model $(570 \mathrm{mT})$ is reduced by less than $10 \%$ with respect to the SMF estimated by the $3 \mathrm{D}$ complete model $(620 \mathrm{mT}) ; 4)$ the SMF generated by the $\mathrm{NdFeB}$ ring magnets does not reduce cell-specific metabolic activity after $48 \mathrm{~h}$ and 7 days in 2D and 3D conditions (both static and dynamic); 5) the SMF exerts a stressful effect in 2D conditions, but it decreases in 3D conditions (both static and dynamic).

Due to the very limited experimental accessibility of our miniaturized system to measurement of the magnetic field, we used a numerical analysis to predict the magnetic field generated by the magnetic rings. In fact, the culture chambers are very small $\left(9 \mathrm{~mm}^{3}\right)$ and it was not possible to find a suitable magnetometer able to avoid any influence on the read-outs while measuring. Moreover, a magnetometer is not able to show the distribution of the magnetic field. Peng and co-

\section{D CONDITIONS}

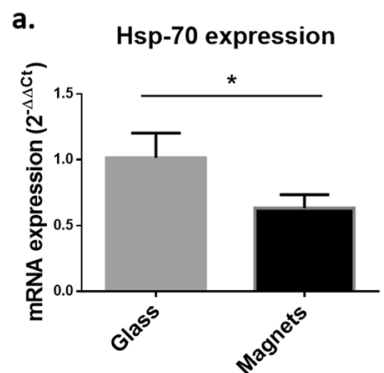

Fig. 7 Influence of the magnetic field on SH-SY5Y cells in 2D culture: mRNA expression levels. a mRNA expression levels of heat shock protein-70 (Hsp70). b a) mRNA expression levels of Bcl-2. c mRNA b.

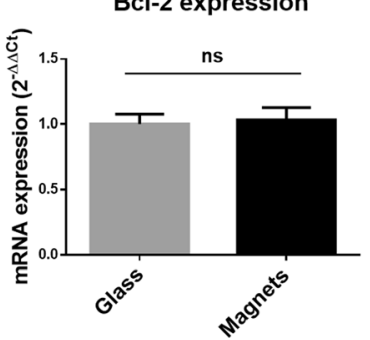

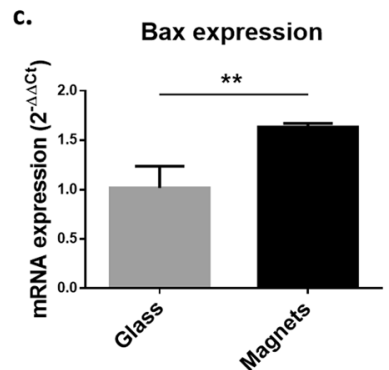

expression levels of Bax. We reported the results as mean $\pm \mathrm{SD}, 3$ replicates/group. We performed the statistical analysis with t-test. ns, $p>0.05$; *, $p<0.05 ; * *, p<0.01$ 

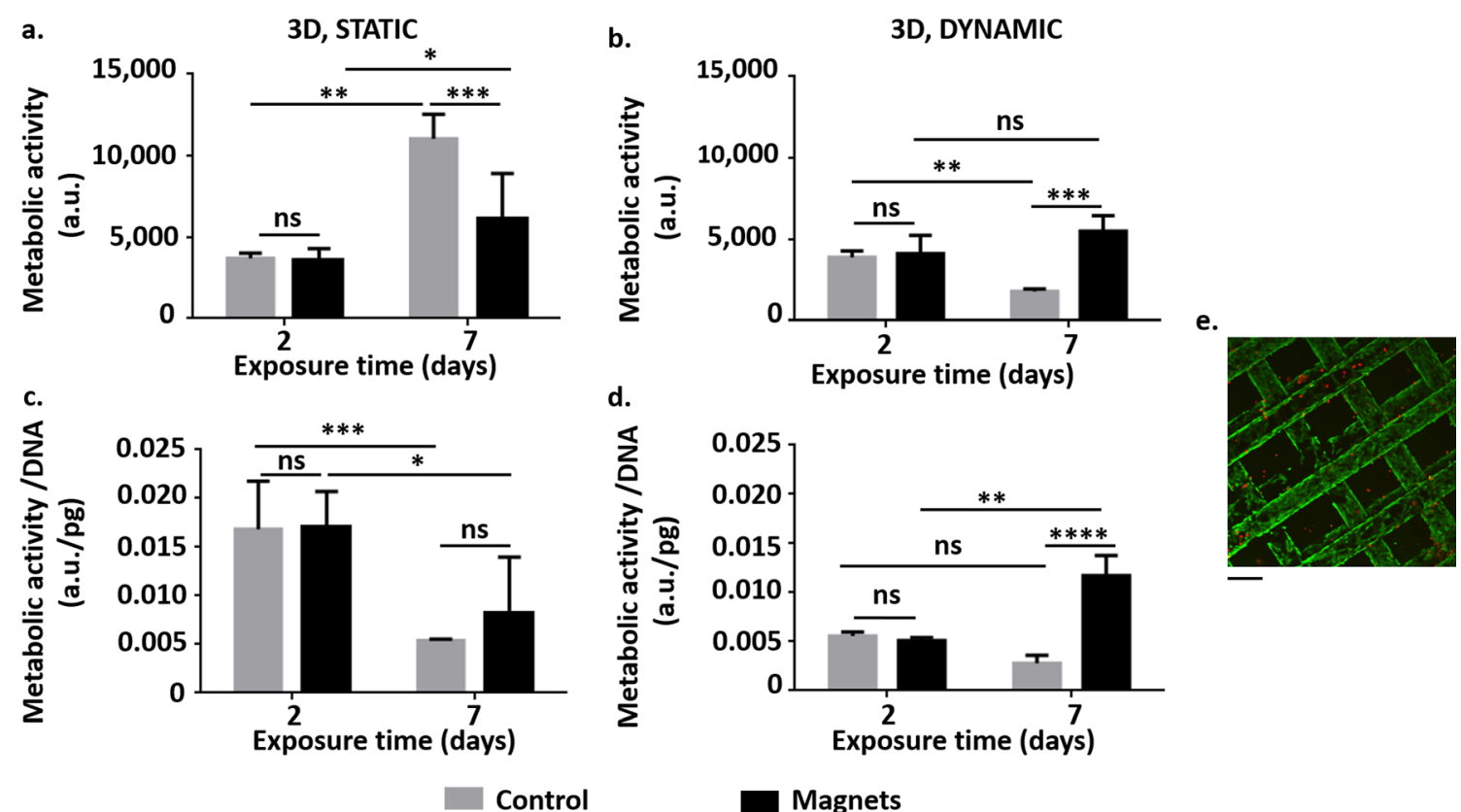

Fig. 8 Influence of the magnetic field on SH-SY5Y cells in 3D culture: specific metabolic activity. a Metabolic activity of SH-SY5Y cells with time (in static conditions). b Metabolic activity of SH-SY5Y cells with time (in dynamic conditions). c Specific metabolic activity (that is metabolic activity normalized with respect to the DNA content) of SHSY5Y cells with time (in static conditions). d Specific metabolic activity of SH-SY5Y cells with time (in dynamic conditions). In all the graphs, we showed the results from resazurin assay (3 replicates/group). We reported

workers described an analytical method to calculate the magnetic field only for the central axis of an axially magnetized ring (Peng et al. 2004), but to our knowledge no analytical methods calculating the distribution of the magnetic field around a magnetic ring exist. Instead of $\mathrm{NdFeB}$, we considered neodymium as the material for the domains of magnetic coupling because it is the strongest magnetic material of the alloy. In fact, neodymium magnets have a coercivity of about four orders of magnitude greater than that of a material with only a ferrous component.

\section{Magnets}

the results as mean $\pm \mathrm{SD}$. We performed the statistical analysis with twoway ANOVA followed by Tukey's multiple comparisons test. ns, $p>0.05 ; *, p<0.05 ; * *, p<0.01 ; * * *, p<0.001 ; * * * *, p<0.0001$. e Example of confocal microscopy images showing live (stained green by calcein AM) and dead (stained red by ethidium homodimer-1) SH-SY5Y cells after $48 \mathrm{~h}$ culturing in static conditions in the absence of the magnetic field. Results from a z-stack acquisition. Scale bar $20 \mu \mathrm{m}$

Taking as a reference the intensity of Earth magnetic field $(25-65 \mu \mathrm{T})$, we estimated greater values $(320-620$ $\mathrm{mT})$ in the geometrical center of the rings. Therefore, the assessment of a possible influence of the magnetic field on cell constructs was necessary.

In the last few years, several studies have focused on the influence of SMFs at the cellular level. They have reported a dependence on magnetic field intensity, exposure time, cell type and density, but results are contradictory and a comparison is difficult because of the differences in experimental

3D CONDITIONS

a.

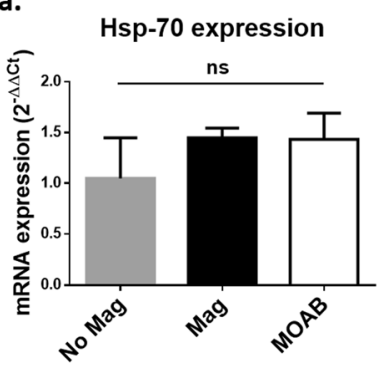

b.

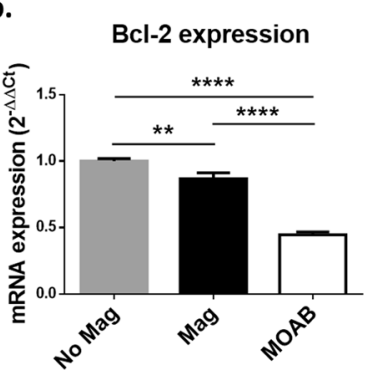

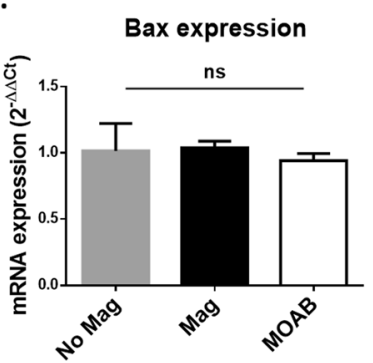

Fig. 9 Influence of the magnetic field on SH-SY5Y cells in 3D culture: mRNA expression levels. a mRNA expression levels of heat shock protein-70 (Hsp70). b a) mRNA expression levels of Bcl-2. c mRNA expression levels of Bax. We reported the results as mean $\pm \mathrm{SD}, 3$ replicates/group. We performed the statistical analysis with one-way ANOVA followed by Dunnett's multiple comparisons test. ns, $p>0.05$; $* * *, p<0.001 ; * * * *, p<0.0001$ 
parameters and read-outs (e.g. cell number, cell metabolic activity, orientation of intracellular components, DNA transcription). Since changes in cell cycle or at intracellular level (e.g. growth factor signaling, DNA transcription) have a direct impact on cell viability and functions (Albuquerque et al. 2016), in this work we focused on cell metabolic activity, number and gene expression. For 2D studies, we preferred dishes with glass bottom to standard polystyrene microplates because the latter does not allow for cell observation by confocal microscopy. Since the multi-layer nickel-copper-nickel plating may not be sufficient to prevent the corrosion of neodymium magnets for all applications, we covered $\mathrm{NdFeB}$ magnets with a protective layer of High Temp Resin after assessing its biocompatibility with SH-SY5Y cells (Online Resource 1).

In both $2 \mathrm{D}$ and $3 \mathrm{D}$ conditions, we observed that the SMF does not reduce cell-specific metabolic activity after $48 \mathrm{~h}$ and 7 days of exposure. For cells in 2D conditions, Miyakoshi (2005) published that most of studies reported no significant effects of SMF on cell viability. Similarly, Romeo and coworkers (Romeo et al. 2016) reported lack of effects on cell viability when exposing MRC-5 human lung fibroblasts to a SMF of $370 \mathrm{mT}$. Oppositely, biological effects were observed when the magnetic field was coupled to stimuli like $\mathrm{x}$-irradiation and in the literature there is evidence that SMFs can affect several endpoints when in the mT range. For SH-SY5Y cells exposed for $24 \mathrm{~h}$ to $2.2 \mathrm{mT}$ SMF, Calabrò and colleagues (Calabrò et al. 2013) observed a decrease of membrane mitochondrial potential up to $30 \%$ and an increase in the production of reactive oxygen species. On the other end, Stolfa and co-workers (Stolfa et al. 2007) reported an increase in viability for human chondrocytes exposed for $72 \mathrm{~h}$ to a SMF of $600 \mathrm{mT}$. To our knowledge, this is the first study examining the effects of the SMF on SH-SY5Y cells in 3D conditions, therefore no direct comparison with data in the literature is possible.

We deepened our investigations with genetic assays and analysed the expression profile of Hsp70, Bcl-2 and Bax. Hsp70 can be activated when cells are exposed to particular stressors and it represents one of the actors of the apoptosis regulation. Bcl-2 is an anti-apoptotic protein that inhibits cell death by regulating the Bcl-2-regulated apoptotic pathway, while Bax has a pro-apoptotic role in the events triggering programmed cell death. We selected these genes from a previous work (Tenuzzo et al. 2009). Here, the authors evaluated the biological effects of a SMF of $6 \mathrm{mT}$ on aged human lymphocytes. Similarly to Tenuzzo and colleagues, we demonstrated that the expression of Hsp70 decreases and the expression of Bax increases after 7day exposure to our SMF in 2D conditions. Together with the results from cell metabolic activity, these results suggest that the SMF generated by the NdFeB ring magnets is not stressful enough to reduce cell-specific metabolic activity. In 3D dynamic conditions (MOAB samples), Hsp70 and Bax expression was comparable with controls (No Mag samples), suggesting a reduction of this stressful effect. The significant lower levels of Bcl-2 in SH-SY5Y cells subjected to the SMF suggested a possible weak stressed condition that should be further investigated.

The hydraulic characterization performed in the present study indicated that both the magnetically and snap-fit lockable MOABs may stand pressure values higher than those usually involved in cell perfusion experiments, where maximum flow rates are in the order of $\mu \mathrm{L} / \mathrm{min}$. However, at higher pressures the flow rate offered by the bioreactor with magnetic closing is significantly greater. This is probably due to the better hydraulic sealing of the magnetic coupling with respect to the simple snap-fit closure for mechanical interference. Cell proliferation inside the scaffolds increases the mechanical resistance to the fluid flow within the chambers. To avoid leakage and guarantee a suitable perfusion to cell constructs during experiments up to 7 days, we inserted screws and cable ties on non-magnetically bioreactors. Despite these precautions, the specific metabolic activity was significantly lower than in magnetic dynamic samples $(p<0.0001)$, suggesting that our snap-fit bioreactors are not suitable for perfusion experiments longer than $48 \mathrm{~h}$. For these reasons, we did not perform genetic assays on these samples.

However, the MOAB with snap-fit closure would remain a forced choice for magnetic particle tracking, apart from their dimensions and charge.

\section{Conclusions}

We predicted the values and distribution of the SMF generated by the $\mathrm{NdFeB}$ ring magnets in the culture chambers of the MOAB by a numerical analysis, indicating that the intensity of the magnetic field has a quadratic decay trend along the radial direction of the culture chamber, with very low values affecting all the central areas of the cultured scaffold. Furthermore, the maximum intensity of the magnetic field generated in each chamber of the bioreactor is not influenced by the adjacent chamber(s). The hydraulic characterization recorded a significantly higher leakage for non-magnetically lockable MOABs compared to the standard magnetic ones. We found that after 2 and 7 days of cell exposure to the SMF generated by the magnetic closure of the MOAB, the cell-specific metabolic activity of SH-SY5Y cells is not reduced, neither in 2D monolayer, nor in $3 \mathrm{D}$ static culture, nor in the MOAB. Instead, the expression levels of Hsp70, Bcl-2 and Bax measured in cells at seven culture days shows that this level of SMF exerts a stressful effect in 2D monolayer, which decreases to a negligible level in $3 \mathrm{D}$ static culture, and in the MOAB. Taken together, these results suggest that MOAB device with magnetic closure might be potentially used also as the basic functional unit of the ERC "MINERVA" multi-organ bioengineered platform. 
Acknowledgments This study was funded by the European Research Council (ERC) under the European Union's Horizon 2020 research and innovation program (Grant agreements No. 724734-MINERVA to C.G. and 825159-MOAB to M.T.R.). The results reflect only the authors' views and the Agency is not responsible for any use that may be made of the information contained. We thank Dr. Alessandro Marturano-Kruik for his technical support, Prof. Giambattista Gruosso, Politecnico di Milano, for his suggestions on the numerical models and Dr. Diego Albani, Istituto di Ricerche Farmacologiche Mario Negri-IRCCS, for critically reviewing the manuscript.

\section{Compliance with ethical standards}

Conflict of interest The authors declare that they have no conflict of interest.

Open Access This article is distributed under the terms of the Creative Commons Attribution 4.0 International License (http:// creativecommons.org/licenses/by/4.0/), which permits unrestricted use, distribution, and reproduction in any medium, provided you give appropriate credit to the original author(s) and the source, provide a link to the Creative Commons license, and indicate if changes were made.

\section{References}

W.W. Albuquerque, R.M. Costa, S. Fernandes Tde, A.L. Porto, Prog. Biophys. Mol. Biol. 121, 16 (2016). https://doi.org/10. 1016/j.pbiomolbio.2016.03.003

C. Aldinucci, J.B. Garcia, M. Palmi, G. Sgaragli, A. Benocci, A. Meini, F. Pessina, C. Rossi, C. Bonechi, G.P. Pessina, Bioelectromagnetics. 24, 6 (2003)

E. Calabrò, S. Condello, M. Currò, N. Ferlazzo, D. Caccamo, S. Magazù, R. Ientile, Bioelectromagnetics. 34, 618 (2013). https://doi.org/10. 1002/bem. 21815

J. Dolezel, J. Bartos, H. Voglmayr, J. Greilhuber, Cytometry A. 51, 2 (2003)

European Commission (EC). Non-ionizing radiation: sources, exposure and health effects. B-1049, Brussels 1996

M. Feychting, Prog. Biophys. Mol. Biol. 87, 2-3 (2005)

P. Frattini, C. Villa, F.D. Santis, M. Meregalli, M. Belicchi, S. Erratico, P. Bella, M.T. Raimondi, Q. Lu, Y. Torrente, Hum. Mol. Genet. 26, 3682 (2017). https://doi.org/10.1093/hmg/ddx252

V. Hartwig, S. Romeo, O. Zeni, Med. Biol. Eng. Comput. 56, 531 (2018). https://doi.org/10.1007/s11517-017-1779-7

W. Ji, H. Huang, A. Deng, C. Pan, Micron. 40, 894 (2009). https://doi. org/10.1016/j.micron.2009.05.010

H.J. Kim, D. Huh, G. Hamilton, D.E. Ingber, Lab Chip 12, 2165 (2012). https://doi.org/10.1039/c2lc40074j
F. Kong, H. Wang, J. Guo, M. Peng, H. Ji, H. Yang, B. Liu, J. Wang, X. Zhang, S. Li, In Vitro Cell Dev Biol Anim. (2016). https://doi.org/ 10.1007/s11626-016-0005-5

M. Laganà, M.T. Raimondi, Biomed. Microdevices 14, 225 (2012). https://doi.org/10.1007/s10544-011-9600-0

K.F. Lei, M.H. Wu, C.W. Hsu, Y.D. Chen, Biosens. Bioelectron. 51, 16 (2014). https://doi.org/10.1016/j.bios.2013.07.031

M. Lübberstedt, U. Müller-Vieira, K.M. Biemel, M. Darnell, S.A. Hoffmann, F. Knöspel, E.C. Wönne, D. Knobeloch, A.K. Nüssler, J.C. Gerlach, T.B. Andersson, K. Zeilinger, J. Tissue Eng. Regen. Med. (2015). https://doi.org/10.1002/term.1652

D. Lv, Z. Hu, L. Lu, H. Lu, X. Xu, Oncol. Lett. (2017). https:// doi.org/10.3892/ol.2017.7134

A. Marturano-Kruik, M.M. Nava, K. Yeager, A. Chramiec, L. Hao, S. Robinson, E. Guo, M.T. Raimondi, G. Vunjak-Novakovic, Proc. Natl. Acad. Sci. U. S. A. 115, 1256 (2018). https://doi.org/10. 1073/pnas.1714282115

J. Miyakoshi, Prog. Biophys. Mol. Biol. 87, 2-3 (2005)

J. Pawlowski, A.S. Kraft, Proc Natl Acad Sci U S A. 97, 2 (2000)

Q.L. Peng, S.M. Mc Murry, J.M.D. Coey, J. Magn. Magn. Mater. 268, 12 (2004)

R.R. Raylman, A.C. Clavo, R.L. Wahl, Bioelectromagnetics. 17, 5 (1996)

S. Romeo, A. Sannino, M.R. Scarfi, R. Massa, R. d'Angelo, O. Zeni, Sci. Rep. 6 (2016). https://doi.org/10.1038/srep19398

B.J. Roth, Exp. Biol. Med. (Maywood). (2011). https://doi.org/10.1258/ ebm.2010.010236

S. Stolfa, M. Skorvánek, P. Stolfa, J. Rosocha, G. Vasko, J. Sabo, Physiol Res. 56(Suppl 1) (2007)

B. Tenuzzo, C. Vergallo, L. Dini, Tissue Cell. 41, 169 (2009). https://doi. org/10.1016/j.tice.2008.09.004

M. Tunesi, F. Fusco, F. Fiordaliso, A. Corbelli, G. Biella, M.T. Raimondi, Front. Aging Neurosci. 8 (2016). https://doi.org/ 10.3389/fnagi.2016.00146

O. Valiron, L. Peris, G. Rikken, A. Schweitzer, Y. Saoudi, C. Remy, D. Job, J. Magn. Reson. Imaging 22, 334 (2005). https://doi.org/10. 1002/jmri.20398

S. Zannella, in CAS-CERN Accelerator School: Measurement and Alignment of Accelerator and Detector Magnets. (1997) https:// doi.org/10.5170/CERN-1998-005.375

L. Zhang, X. Ji, X. Yang, X. Zhang, Oncotarget. (2017). https://doi.org/ 10.18632/oncotarget.14480

Publisher's note Springer Nature remains neutral with regard to jurisdictional claims in published maps and institutional affiliations. 